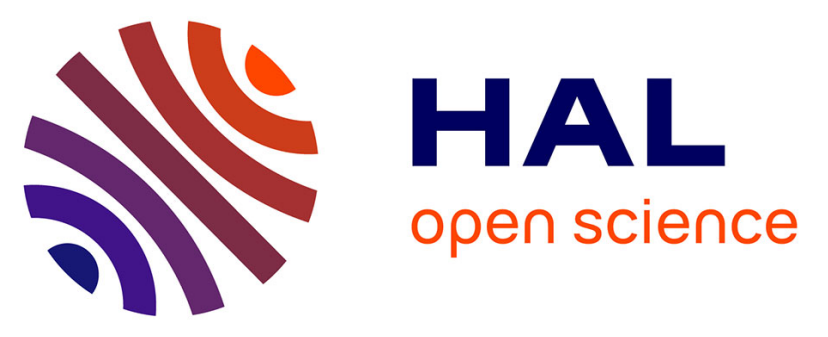

\title{
Increased sensitivity of a new commercial reverse transcriptase-quantitative PCR for the detection of Pneumocystis jirovecii in respiratory specimens
}

Sarah Dellière, Samia Hamane, Nesrine Aissaoui, Maud Gits-Muselli, Stéphane Bretagne, Alexandre Alanio

\section{To cite this version:}

Sarah Dellière, Samia Hamane, Nesrine Aissaoui, Maud Gits-Muselli, Stéphane Bretagne, et al.. Increased sensitivity of a new commercial reverse transcriptase-quantitative PCR for the detection of Pneumocystis jirovecii in respiratory specimens. Medical Mycology, 2021, pp.myab029. 10.1093/mmy/myab029 . pasteur-03226313

\section{HAL Id: pasteur-03226313}

https://hal-pasteur.archives-ouvertes.fr/pasteur-03226313

Submitted on 14 May 2021

HAL is a multi-disciplinary open access archive for the deposit and dissemination of scientific research documents, whether they are published or not. The documents may come from teaching and research institutions in France or abroad, or from public or private research centers.
L'archive ouverte pluridisciplinaire HAL, est destinée au dépôt et à la diffusion de documents scientifiques de niveau recherche, publiés ou non, émanant des établissements d'enseignement et de recherche français ou étrangers, des laboratoires publics ou privés.

\section{(ㄷ)(1) $\$$}

Distributed under a Creative Commons Attribution - NonCommerciall 4.0 International 
1 Increased sensitivity of a new commercial reverse transcriptase-

2 quantitative PCR for the detection of Pneumocystis jirovecii in respiratory

4

5 Sarah Dellière ${ }^{1,2}$, Samia Hamane ${ }^{1}$, Nesrine Aissaoui ${ }^{1}$, Maud Gits-Muselli ${ }^{1,2}$, Stéphane Bretagne ${ }^{1,2,3}$,

Alexandre Alanio ${ }^{1,2,3}$

7

$8 \quad{ }^{1}$ Université de Paris, Laboratoire de Parasitologie-Mycologie, Groupe Hospitalier Saint-Louis-Lariboisière-

9 Fernand-Widal, Assistance Publique-Hôpitaux de Paris (AP-HP), Paris, France.

$10 \quad{ }^{2}$ Institut Pasteur, Molecular Mycology Unit, CNRS UMR2000

$11{ }^{3}$ National Reference Center for Invasive Mycoses and Antifungals (NRCMA), Institut Pasteur, Paris, France 12

13 ** Corresponding author. Mailing address: Alexandre Alanio, Molecular Mycology unit, Institut Pasteur, 25 rue 14 du Dr Roux 75724 Paris Cedex 15; email: alexandre.alanio@pasteur.fr; Tel: +33140613255; Fax:

$15+33145688420$

16

17 Keyword : Pneumocystis jirovecii, real time PCR, diagnosis, Pneumocystis pneumonia, mitochondria 


\section{Abstract}

20 Optimal sensitivity to detect low Pneumocystis loads is of importance to take individual and collective 21 measures to avoid evolution towards Pneumocystis pneumonia and outbreaks in immunocompromised 22 patients. This study compares two qPCR procedures, a new automated RTqPCR using the GeneLEAD 23 VIII extractor/thermocycler (GLVIII; 2.2 hrs workflow) and a previously validated in-house qPCR 24 assays (IH; 5 hrs workflow) both targeting mtSSU and mtLSU for detecting P. jirovecii in 213 25 respiratory samples. GLVIII was found to be more sensitive than $\mathrm{IH}$, detecting 8 more specimens. 26 Bland-Altman analysis between the two procedures showed a Cq bias of $1.17 \pm 0.07$ in favor of GLVIII.

\section{Lay summary}

30 The fungus Pneumocystis needs to be detected early in respiratory samples to prevent pneumonia in 31 immunocompromised hosts. We evaluated a new commercial RTqPCR on 213 respiratory samples to 32 detect Pneumocystis and found it more sensitive and faster than our routine sensitive in-house qPCR 33 assay. 

pneumonia (PCP). ${ }^{1}$ PCP diagnosis relies on X-ray showing bilateral or diffuse ground-glass opacities with interstitial infiltrates as a predominant feature, microscopy of respiratory samples and more and more often on detection of nucleic acids in respiratory samples. ${ }^{2}$ Furthermore, the good negative predictive value of serum B-D-glucan is used as an additional tool to rule out PCP or increase the confidence in the diagnosis when positive. ${ }^{3}$ Optimal sensitivity to detect low fungal load is of major importance to avoid progression towards PCP and preventing cross-contamination between immunocompromised patients in taking appropriate measures such as cotrimoxazole prophylaxis. ${ }^{2,4} \mathrm{An}$ international initiative has recently demonstrated a better sensitivity of reverse transcriptase quantitative PCR (RTqPCR) methods, amplifying whole nucleic acid (WNA, including DNA and RNA) and targeting the mitochondrial small subunit of rRNA gene (mtSSU) over other qPCR methods and targets. ${ }^{5}$ We compare here two qPCR procedures both incorporating validation internal controls (DNA or RNA alien target, DIA-EIC/DNA-050/ DIA-EIC/RNA-050 for qPCR and RTqPCR assays), ${ }^{6}$ a new fully automated RTqPCR ( 2.2 hrs workflow) and our in-house qPCR assays ( $\sim 5$ hrs workflow: sample preparation and pre-extraction step $\sim 30 \mathrm{~min}$, extraction $\sim 1 \mathrm{hrs} 30 \mathrm{~min}$, qPCR mix and plate preparation $\sim 45 \mathrm{~min}, \mathrm{qPCR} \sim 2 \mathrm{hrs}$ ) both targeting $\mathrm{mtSSU}$ and $\mathrm{mtLSU}$ for detecting $P$. jirovecii in respiratory samples including bronchoalveolar lavage fluids (BAL) and non-BAL specimens. Of note both assays were performed using 45 cycles.

Upon reception, samples were centrifuged and pellet was resuspended in $400 \mu \mathrm{L}$ and divided in two equal parts. Mucous specimens (mucous BAL, bronchial aspirates, or sputa) were treated with $1 \mathrm{X}$ dithiothreitol (dithiothreitol, dTT, digest-EUR, EuroBio) for $15 \mathrm{~min}$ at $37^{\circ} \mathrm{C}$, centrifuged with the supernatant discarded. To our knowledge, dTT does not impact negatively the results of qPCR, as it is recommended in lysis buffer and used in extraction buffers. The tested procedure included WNA extraction using the GeneLEAD VIII extractor-thermocycler (Precision System Science, Japan) and the new R-DiaPnJ kit (Diagenode, Seraing, Belgium) amplifying mtLSU and mtSSU WNA (designated as

60 GLVIII). The reference procedure included WNA extraction using the DSP virus/Pathogen kit on a 61 QIAsymphony apparatus (Qiagen, Hilden, Germany) and amplification using our in-house mtSSU and mtLSU qPCR assays (designated as IH). ${ }^{7}$ This non-interventional study on leftover specimens did not 
require approval of an ethics committee according to the French Health Public Law (CSP Art L1121$1.1)$.

Full validation of the GLVIII was performed and characteristics are available in Table S1. A total of 213 consecutive respiratory samples were tested from 167 patients described in Table 1 . Pneumocystis jirovecii nucleic acids were detected in 45 specimens regardless of the PCR procedure. A total of 44 samples were positive with GLVIII and 37 with IH, with 36 samples positive with both methods. Four specimens ( 2 BAL and 2 non-BAL samples) were deemed invalid based on internal control in the GLVIII procedure with all four being negative using the IH procedure. Agreement between both techniques was $>0.81$ with a Cohen's kappa $=0.863)$ (Table S2). For GLVIII procedure, 40/44 (90.91\%) were positive with both mtLSU and mtSSU targets, 2/44 with mtLSU only, and 2/44 with mtSSU only.

Eight samples from eight patients were GLVIII-positive only with mean quantification cycle (Cq) of $35.1( \pm 0.5)$ for mtLSU and of $34.6( \pm 1.3)$ for mtSSU targets. The only IH-positive and GVIIInegative sample was not validated by the GLVIII controls, although no PCR inhibitors were evidenced with the IH internal control. All patients' characteristics with discrepancies between GVIII and IH are shown in Table S3. None received PCP prophylaxis. Overall, the Cq values obtained in the 36 samples positive in both methods were significantly lower with the GLVIII than with the IH (mean Cq difference was $-3.9 \pm 1.7$ for mtLSU and $-2.5 \pm 2.2$ for $\mathrm{mtSSU}$ ) ( $\mathrm{p}<0.001)$. Bland-Altman analysis between the two procedures showed a Cq bias of $1.17 \pm 0.07$ in favor of GLVIII. For specific analysis, redundant sampling per patients were excluded and analysis was replicated in BAL (not treated with dTT, $n=23$; Figure 1A and 1C) and non-BAL specimens (treated with dTT, n=16; Figure 1B and 1D), separately. Quantification cycle distributions were significantly different in all four assays (non-parametric paired Anova test, $\mathrm{p}<0.0001)$. Mean Cq difference between IH and GLVIII was $-4.2 \pm 1.8$ for mtLSU and -3.7 \pm 1.8 for $\mathrm{mtSSU}$, for BAL specimens, and $-3.9 \pm 1.5$ for $\mathrm{mtLSU}$ and $-1.6 \pm 2.4$ for $\mathrm{mtSSU}$, for nonBAL specimens.

Despite that the R-DiaPnJ kit was validated only on bronchoalveolar lavage fluids, we performed our study regardless of the specimen type. We finally analyzed 90 non-BAL specimens (37 bronchial 
aspirate and 53 sputa). A total of 14/16 qPCR-positive specimens (87.5\%) were positive with both procedures and two were positive with only one of both assays (1 with GLVIII only and 1 with IH only). GLVIII mtLSU target was the most frequently positive target gene (15/16) as compared to GLVIII mtSSU target (14/16 positive.)

Since the PCR targets were identical, differences result potentially from several factors including extraction method, amplification of WNA versus DNA only, platform and mastermix as already described. ${ }^{6}$ Indeed WNA versus DNA extraction and amplification may result in a $\Delta$ Cq of 5.8 for low fungal loads as previously shown. ${ }^{6}$ Depending on master mix selected, Cq may also vary with a $\Delta \mathrm{Cq}$ up to 2.9 between two master mixes with otherwise identical extraction and amplification protocol. ${ }^{6}$ The use of two targets in this commercial assay is a significant asset because it strengthens data analysis with a more confident result in case both targets are positive. It also prevents complete nondetection due to mutations in one of the target sequences preventing proper primers/probe annealing. ${ }^{8}$ As an example, detection of SARS-CoV-2 variant with mutations on the spike protein could only be performed by multiplex PCR assays targeting other genes. ${ }^{9}$ Furthermore, the manufacturer has disclosed that the mtSSU probe had a Minor Groove Binder (MGB) quencher. MGB probes were designed to increased specificity of detection especially for single base mismatches at elevated hybridization temperatures..$^{10}$ A previous Pneumocystis qPCR assay using a MBG probe was confronted with false negative results due to an undescribed mutation of the probe hybridization region in the mtLSU gene with an estimated frequency of $0.28 \%{ }^{8}$

As part of a previous international initiative, our center compared on the same qPCR thermocycler 10 assays including in-house $(n=5)$ and commercial $(n=5)$ assays. ${ }^{5}$ When our in-house mtLSU qPCR is used as reference to compare all tested assays, GLVIII (mtSSU target) comes in the second $(\Delta \mathrm{Cq}$ of $-4.7 \pm 1.8)$ and third position $(-3.9 \pm 1.7)$ just after an in-house RT-qPCR targeting mtSSU with a $\Delta \mathrm{Cq}$ of $-6.6( \pm 0.8)^{7}$ and before the other commercial assays evaluated (Table S4). GLVIII procedure, giving better detection rate and $\mathrm{Cq}$ results, seems to be a good assay to quantify more accurately the fungal load in respiratory samples. Indeed, semi-quantification allows clinical interpretation of fungal loads with high fungal loads associated with PCP and low fungal load to carriage which may require prophylaxis for both individual and collective prevention in immunocompromised 
118 populations. ${ }^{2,11,12}$ A prospective clinical study is required to properly determine clinical cut-offs with 119 this assay.

120 In conclusion, we report here an increased sensitivity using a new molecular diagnostic 121 procedure compared to our in-house procedure. ${ }^{11}$ In addition to a better sensitivity, the diagnosis is 122 achieved within $\sim 2$ hours upon reception of the sample using this new procedure. The GeneLEAD

123 VIII/R-DiaPnJ kit is a single sample assay which is clinically relevant when accurate sensitive detection 124 and prompt therapeutic decision are at stake.

125

Authors contribution

Writing - Original draft: SD and AA; Writing - Review and editing: All; Conceptualization: AA;

Formal Analysis: SD and AA; Supervision: AA

\section{Conflict of Interest}

133 AA and SB are owner of a patent on Pneumocystis jirovecii diagnosis using RTqPCR

\section{References}

136 1. Thomas CF, Limper AH. Pneumocystis pneumonia. N Engl J Med. 2004;350(24):2487-2498. doi:10.1056/NEJMra032588.

2. Alanio A, Hauser PM, Lagrou K, et al. ECIL guidelines for the diagnosis of Pneumocystis jirovecii pneumonia in patients with haematological malignancies and stem cell transplant recipients. The Journal of Antimicrobial Chemotherapy. 2016;71(9):2386-2396. doi:10.1093/jac/dkw156.

142 3. Lagrou K, Chen S, Masur H, et al. Pneumocystis jirovecii Disease: Basis for the Revised EORTC/MSGERC Invasive Fungal Disease Definitions in Individuals Without Human Immunodeficiency Virus. Clin Infect Dis. 2021;72(Supplement_2):S114-S120.

146 4. Dellière S, Gits-Muselli M, Bretagne S, Alanio A. Outbreak-Causing Fungi: Pneumocystis jirovecii. Mycopathologia. 2020;185(5):783-800. doi:10.1007/s11046-019-00408-w.

148 5. Gits-Muselli M, White PL, Mengoli C, et al. The Fungal PCR Initiative's evaluation of in- 
6. Dellière S, Gits-Muselli M, White PL, Mengoli C, Bretagne S, Alanio A. Quantification of Pneumocystis jirovecii: Cross-Platform Comparison of One qPCR Assay with Leading Platforms and Six Master Mixes. J Fungi. 2020;6(1):9-9. doi:10.3390/jof6010009.

7. Valero C, Buitrago MJ, Gits-Muselli M, et al. Copy Number Variation of Mitochondrial DNA Genes in Pneumocystis jirovecii According to the Fungal Load in BAL Specimens. Frontiers in Microbiology. 2016;7(733):237-11. doi:10.3389/fmicb.2016.01413.

8. Le Gal S, Robert-Gangneux F, Pépino Y, et al. A misleading false-negative result of Pneumocystisreal-time PCR assay due to a rare punctual mutation: A French multicenter study. Medical Mycology. 2017;55(2):180-184. doi:10.1093/mmy/myw051.

9. Bal A, Destras G, Gaymard A, et al. Two-step strategy for the identification of SARS-CoV-2 variant of concern 202012/01 and other variants with spike deletion H69-V70, France, August to December 2020. Euro Surveill. 2021;26(3):960. doi:10.2807/15607917.ES.2021.26.3.2100008.

164

10. Kutyavin IV, Afonina IA, Mills A, et al. 3'-minor groove binder-DNA probes increase sequence specificity at PCR extension temperatures. Nucleic Acids Research. 2000;28(2):655661. doi:10.1093/nar/28.2.655.

11. Alanio A, Desoubeaux G, Sarfati C, et al. Real-time PCR assay-based strategy for differentiation between active Pneumocystis jirovecii pneumonia and colonization in immunocompromised patients. Clin Microbiol Infect. 2011;17(10):1531-1537. doi:10.1111/j.1469-0691.2010.03400.x.

12. Alanio A, Bretagne S. Pneumocystis jirovecii detection in asymptomatic patients: what does its 
177 Table 1. Patient and sample characteristics.

\begin{tabular}{|l|c|c|}
\hline & Patients $(\mathrm{n}=167)$ & Samples (n=213) \\
\hline Male $n(\%)$ & $112(67.1)$ & \\
\hline Age median [IQR: $Q 1-Q 3]$ & $59[44-67]$ & $123(57.8)$ \\
\hline Type of respiratory sample & & $52(24.4)$ \\
\hline BAL $n(\%)$ & & $38(17.8)$ \\
\hline Sputum $n(\%)$ & & $35(16.4)$ \\
\hline Bronchial aspirate $n(\%)$ & $39(17.4)$ & $35(16.4)$ \\
\hline P. jirovecii detection rate & $31(18.6)$ & $37(17.4)$ \\
\hline in-house mtLSU & $36(21.6)$ & $42(19.7)$ \\
\hline in-house mtSSU & $36(21.6)$ & $42(19.7)$ \\
\hline in-house any target & $38(22.8)$ & $44(20.7)$ \\
\hline GeneLEAD VIII mtLSU & $4(2.4)$ & $4(1.9)$ \\
\hline GeneLEAD VIII mtSSU & & \\
\hline GeneLEAD VIII any target & & \\
\hline GeneLEAD VIII invalid & & \\
\hline
\end{tabular}

178 BAL: bronchoalveolar lavage; mtLSU, mitochondrial large subunit; mtSSU, mitochondrial small 179 subunit 
182 Figure 1. A. Pneumocystis jirovecii fungal load (Cq values) according to PCR target and protocol tested

183 for BAL (A) and non-BAL (B) samples. Bland-Altman test between in-house mtLSU DNA and 184 GeneLEAD VIII mtSSU WNA Cq values for BAL (C) and non-BAL (D) samples. MtLSU, 185 mitochondrial large subunit; MtSSU, mitochondrial small subunit; WNA, whole nucleic acids. **** $186 \mathrm{p}<0.0001$ (Wilcoxon non-parametric test).
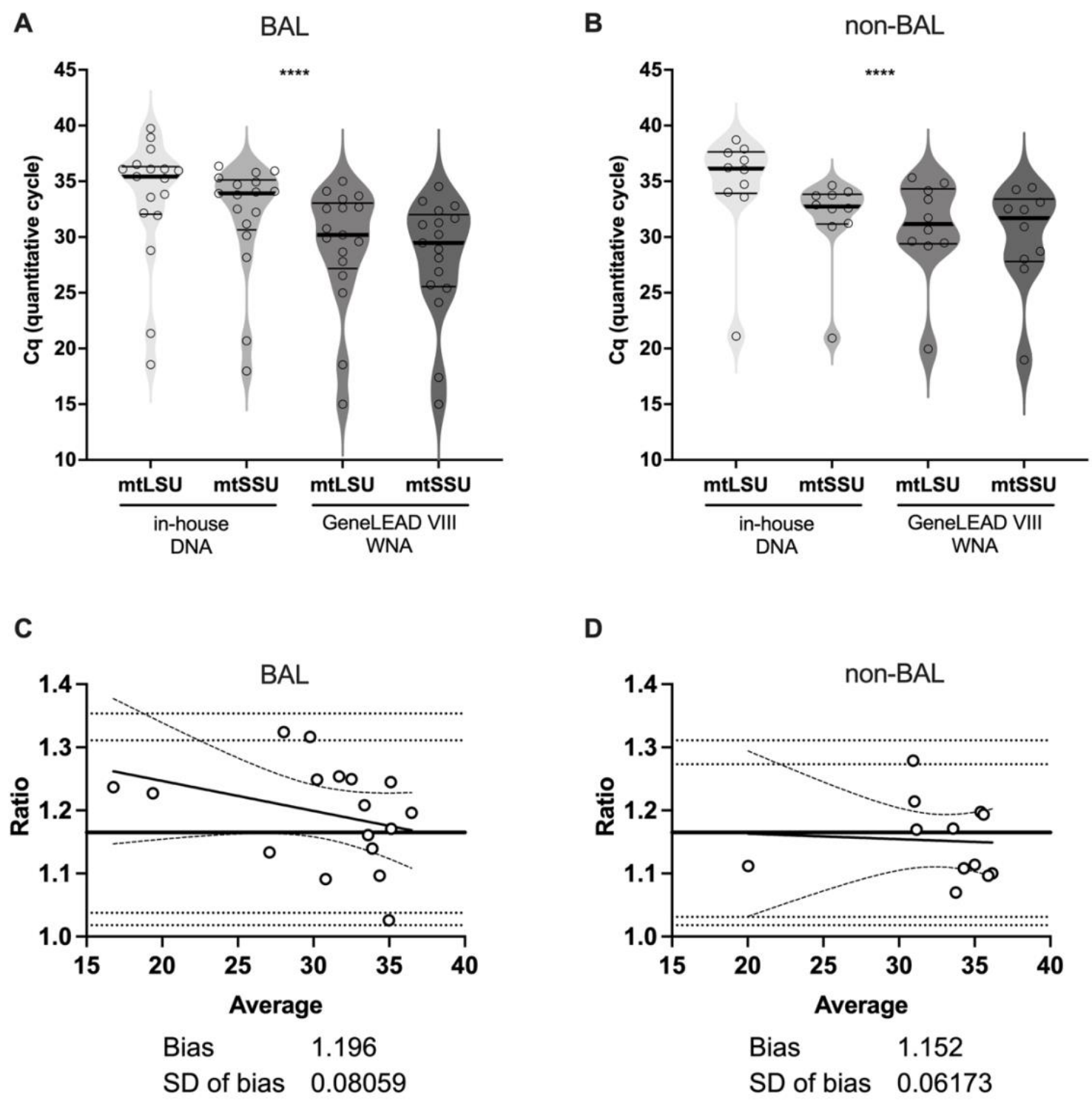